\title{
BAP1: role in carcinogenesis and clinical implications
}

\author{
Daniel A. Kobrinski ${ }^{1}$, Haining Yang $^{2}$, Muaiad Kittaneh ${ }^{1}$ \\ ${ }^{1}$ Department of Oncology, Loyola University Chicago, Maywood, IL, USA; ${ }^{2}$ Department of Cancer Biology, University of Hawaii Cancer Center, \\ Honolulu, HI, USA \\ Contributions: (I) Conception and design: All authors; (II) Administrative support: All authors; (III) Provision of study materials or patients: All \\ authors; (IV) Collection and assembly of data: All authors; (V) Data analysis and interpretation: All authors; (VI) Manuscript writing: All authors; (VII) \\ Final approval of manuscript: All authors. \\ Correspondence to: Muaiad Kittaneh, MD, MBA, FACP. Department of Oncology, Loyola University Chicago, Bldg. 112, Room 270, 2160 S. First \\ Ave, Maywood, IL 60153, USA. Email: mkittaneh@me.com.
}

\begin{abstract}
BRCA-1 associated protein $1(B A P 1)$ is a tumor suppressor gene that has been implicated in the pathogenesis of several malignancies and is gaining more recognition as a crucial gene that could be amenable to therapeutic manipulation. There is a growing interest in prevention and establishing cancer screening guidelines in patients with germline $B A P 1$ mutations to aid with early detection and treatment of associated malignancies. We aim to give a concise review of $B A P 1$ and its role in carcinogenesis, the BAP1 cancer predisposition syndrome and discuss clinical implications including suggested screening guidelines, and potential targeted therapeutic options.
\end{abstract}

Keywords: BRCA-1 associated protein 1 (BAP1); germline; cancer; mesothelioma; genetics

Submitted Sep 02, 2019. Accepted for publication Nov 20, 2019.

doi: $10.21037 /$ tlcr.2019.11.24

View this article at: http://dx.doi.org/10.21037/tlcr.2019.11.24

\section{Introduction}

BRCA-1 associated protein 1 (BAP1) is a tumor suppressor gene of which germline mutations lead to predisposition of several malignancies. The BAP1 gene, located on chromosome 3 p21.1, was discovered by Jensen and colleagues in 1998 (1). BAP1 encodes for a multi-domain protein, BAP1 protein, which contains a $\mathrm{N}$-terminal ubiquitin carboxyl hydrolase (UCH) domain, a host cell factor 1 (HCF-1) binding motif (HBM) domain, and a variety of other binding regions for different protein interactions $(1,2)$. BAP1 functions as a tumor suppressor deubiquitinating enzyme, modulating gene transcription, cellular differentiation, and DNA damage repair, apoptosis and cell metabolism (3-5). Through genomic analyses of family members with both uveal melanoma (UM) and malignant mesothelioma (MM), two rare and unrelated malignancies, a cancer predisposition syndrome implicating germline mutations in BAP1 was discovered and was named as BAP1 tumor predisposition syndrome (BAP1TPDS) (3). Germline BAP1 inactivation is an autosomal dominant disease with high penetrance and increases susceptibility to MM, cutaneous melanoma, UM, clear cell renal cell carcinoma (ccRCC), cholangiocarcinoma, basal cell carcinoma, and likely other malignancies $(3,6,7)$. There has been increased interest in the mechanisms by which BAP1 mutations lead to carcinogenesis. Recently, screening guidelines have been proposed for patients harboring germline BAP1 mutations to aid with early cancer detection. We aim to give a concise review of the carcinogenesis caused by BAP1 mutations, and discuss clinical implications including suggested screening guidelines for patients with germline BAP1 mutations, and potential targeted therapeutic options in patients with mutated $B A P 1$.

\section{BAP1 and carcinogenesis}

BAP1 interacts with proteins that regulate gene expression, such as HCF-1, O-linked $\mathrm{N}$-acetylglucosamine transferase (OGT), and the polycomb group proteins ASXL1 and ASXL2 (8). BAP1 is required for embryogenesis, evident by $B A P 1^{-/-}$murine embryos showing developmental 
retardation at embryonic day 8.5 and subsequent embryonal death (8). Loss of BAP1 in hematopoietic cells induces a phenotype consistent with myelodysplasia and ineffective erythropoiesis (8).

Following DNA damage by ultraviolet (UV) light, there is an increase in BAP1 phosphorylation (9). BAP1 deubiquitylates Ub-H2AK119 at sites of DNA damage, and the loss of BAP1 has been shown to impair homologous recombination (HR) repair (10). The recruitment of BAP1 to sites of UV-induced DNA damage was found to be dependent on poly (ADP ribose) polymerase 1 and 2 (PARP1 and PARP2) (10). BAP1 also controls the accumulation of other DNA-repair proteins (BRCA1, RAD51, and RPA) at sites of DNA damage (10). Given these experimental findings, it is not surprising that a BAP1-mutated RCC cell line treated with a PARP inhibitor, olaparib, showed decreased cell viability (11). Moreover, malignant pleural mesothelioma (MPM) cell lines expressing a BAP1 splice variant with defective deubiquitinating activity treated with olaparib also showed markedly decreased cell viability (12). Clinical trials are underway to investigate the role of PARP inhibitors in patients with DNA-repair protein defects, including BAP1. Currently there is one trial of niraparib (PARP inhibitor) (Clinicaltrials.gov ID NCT03207347) and three trials investigating olaparib (another PARP inhibitor) in BAP 1 and other DNA damage response (DDR) deficient neoplasms (Clinicaltrials.gov ID NCT03786796, NCT03531840, NCT03375307).

Mutations in BAP1 most often affect its catalytic activity (10). Components of polycomb repressive complex 2 (PRC2) are overexpressed in various malignancies such as melanoma, lymphoma, breast and prostate cancer (13). Loss of BAP1 results in accumulation of methylated histone H3 lysine 27 (H3K27) and suppression of PRC2 targets (14). Enhancer of zeste homolog 2 (EZH2) is a protein component of the PRC2 involved in chromatin modification and is one of the two essential catalytic enzymes for the methylation H3K27 in mammalian cells (14). Analysis of The Cancer Genome Atlas (TCGA) data revealed EZH2 mRNA expression was increased in mesothelioma tumor samples compared to matched controls (15). Silencing EZH2 induced apoptosis in BAP 1 -mutant mesothelioma cell lines (15). EZH2 inhibition also reduced mesothelioma tumor size in BAP1-mutant mice (15). In contrast, Schoumacher and colleagues showed EZH2 was not overexpressed in UM cases, and subsequently UM cases with BAP1 loss were insensitive to the EZH2 inhibitor, EPZ-6438 (16). These findings highlight the tissuedependent expression of epigenetic regulators and differing roles in carcinogenesis. Tazemetostat (an EZH2 inhibitor) has been tested in mesothelioma patients with BAP1 lossof-function and shoed some promising activity. The disease control of tazemetostat was $51 \%$ at 12 weeks and $25 \%$ of patients-maintained disease control at 24 weeks (17).

Recent studies have described that cells from $B A P 1$ mutant carriers constitutionally derive a large part of their energy through aerobic glycolysis (18). The Warburg effect is a phenomenon in which cancer cells adapt to an anaerobic environment to promote cell growth (18). Cancer cells, thus, acquire the same amount of energy from fermentation as from the tricarboxylic acid (TCA) cycle (18). Normal fibroblast cell cultures in individuals with heterozygous loss of $B A P 1$ exhibited increased glycolysis and decreased aerobic mitochondrial respiration (5). There were significant differences in glycolysis and the signature of TCA cycle metabolites between BAP1 wild-type (WT) and BAP1 mutant fibroblast cell cultures (5). The Warburg effect promotes carcinogenesis by altering cell metabolism in both tumor cells and surrounding stromal cells (19). Consistent with this observation, germline mutations in BAP1 alter cellular metabolism and tumor microenvironment promoting malignant transformation of cells (18). In another report, BAP1 was found to modulate endoplasmic reticulum (ER)-mitochondria calcium release (4). BAP1 was found to localize to the ER where it regulates $\mathrm{Ca}^{2+}$ release by binding to the type 3 inositol 1,4,5 triphosphate receptor (IP3R3), promoting apoptosis (4). DNA damaged cells appear to be particularly susceptible to cancer transformation due to decreased apoptosis in individuals with deficient BAP1 (4).

\section{BAP1 cancer syndrome}

The majority ( $>95 \%$ ) of BAP1 inactivated tumors are due to sporadic mutations in the gene. Since the report of germline mutations predisposing to mesothelioma and UM in two large and distant families (W from Wisconsin and L from Louisiana) (20), there has been a growing interest in reporting detailed family pedigrees with known BAP1 mutations and associated cancer susceptibilities. The average age of onset of various malignancies, including mesothelioma, in 8 different families with known germline BAP1 mutations ranged from 40-60 years (21). Similar results were found in a study of 79 patients selected on the 
basis of specific criteria related to family history of cancer and diagnosis at younger age. Within this cohort, 13 out of 33 patients with diagnosis of mesothelioma before the age of 50 years carried germline $B A P 1$ mutations and 12 of them had an extensive family history of malignancies associated with the BAP1 cancer syndrome. Interestingly, these patients had a significantly improved survival over sporadic mesothelioma cases (22). Most cancers associated with inactivation of $B A P 1$ occur at ages earlier than those seen in sporadic cases, and some as early as teenage years (23). The majority of patients with germline BAP1 mutations have been diagnosed with at least one malignancy (23). The spectrum of tumors observed in BAP1-mutant murine models was similar irrespective of the type or location of the BAP1 mutation (24). Below we discuss the data surrounding the most commonly diagnosed malignancies in individuals with germline BAP1 mutations which include MM, cutaneous melanoma, UM, and ccRCC.

\section{Malignant mesothelioma}

MM originates from mesothelial cells of the pleura (80-90\%), peritoneum (10-15\%) or rarely the pericardium $(<5 \%)(25,26)$. High rates of non-synonymous mutations in BAP1 were found in MPM tumor samples (27). The same study showed $42 \%$ of MPM tumors harbored either $B A P 1$ loss, mutation or both. BAP1 deleterious germline mutations have been reported with varying frequency in patients with $M M$ ranging from 3-31\% $(22,28,29)$. The median age at diagnosis of MM was 54.5 years in patients with germline $B A P 1$ mutations, which is younger than patients with WT BAP1. The anatomical distribution of $\mathrm{MM}$ in individuals with germline $B A P 1$ mutations is different than sporadic cases. Approximately one-half of mesothelioma in BAP1 mutated individuals originates in the pleura and the other half manifest with peritoneal disease (30). Sporadic mesotheliomas, on the other hand, are more frequently diagnosed with pleural rather than peritoneal primary (30).

BAP1 mutant mice have a shortened survival compared to WT mice after exposure to asbestos $(31,32)$. Survival difference could be attributed to earlier development of mesothelioma and death in BAP1 mutant mice after asbestos exposure. Investigators showed that $B A P 1^{+-}$mice compared with BAP1 WT mice exposed to asbestos had increased susceptibility of MM ( $73 \%$ vs. $32 \%$, respectively) leading to decreased survival (31). Mice carrying germline $B A P 1^{+/-}$developed mesothelioma after exposure to doses of asbestos about ten-times lower doses than those inducing mesothelioma in WT mice (32). Observational studies in humans have demonstrated the opposite in terms of survival. Individuals with $B A P 1$ mutations and MM have a significantly increased median overall survival (OS) (60 months) compared to those WT BAP1 individuals (17 months) (28). Baumann et al. showed that median OS was 5 years in 23 patients with $M M$ and germline $B A P 1$ mutations (30). Screening for BAP1 germline mutations should be considered in patients with $M M$ who have a family history of MM, UM, cutaneous melanoma, RCC, cholangiocarcinoma, basal cell carcinoma, and/or a high family incidence of multiple cancers (33). Screening can also be considered in patients with MM who are found to have melanocytic BAP1-mutated atypical intradermal tumors (MBAITs) (33). There are currently no established guidelines for cancer screening in BAP1 mutation carriers other consensus and multidisciplinary expert panel recommendations $(34,35)$. These recommendations are already being used in clinical practice to screen patients and family of patients with $B A P 1$ germline mutations (36).

\section{Suggested screening tests for MM in germline BAP1 mutated individuals (34)}

Begin annual abdominal and respiratory physical examinations starting at 30 years of age. Imaging surveillance using ultrasound (US) or magnetic resonance imaging (MRI) of the chest and/or abdomen can be considered for asymptomatic patients between ages 30-55 every 2 years. In those individuals $>55$ years of age, consider either computed tomography (CT) chest/abdomen or MRI chest/abdomen with intravenous (IV) contrast every 2 years. A diagnostic fluorodeoxyglucose (FDG) positron emission tomography (PET)/CT is not recommended as first-line imaging modality due a higher ionizing radiation dose. If cost is not prohibitive, a renal and chest US can be considered in the years between CT or MRI.

\section{Uveal melanoma}

Gene expression profiling has classified UMs into two main types: low (class 1) and high (class 2) metastatic potential (37). Class 2 tumors contain dedifferentiated cells and have a primitive neuroectodermal cells gene signature (38). There is a high prevalence of somatic BAP1 mutations in class 2 UMs (39). Eighty-four percent of UMs sampled by Harbour and colleagues showed BAP1 mutations (39). 
Germline mutations in BAP1 were discovered in 3/53 (5.7\%) of cases of UM (40). Age of onset was much younger in individuals with germline $B A P 1$ mutations compared to BAP1 WT individuals (39).

\section{Suggested screening tests for UM in germline BAP1 mutated individuals (34)}

An annual ophthalmic exam by a UM specialist should begin at age 16 and biannual exams after age 30 years is recommended, consisting of direct and indirect ophthalmoscopy, fundus photography, and ocular US. Exams starting at a younger age should be considered if there is a family history of UM.

\section{Melanocytic tumors and cutaneous melanoma}

A new melanocytic neoplasm was described by Wiesner and colleagues with an autosomal dominant inheritance pattern in two unrelated families (34). All affected family members developed skin-colored, dome-shaped, well-circumscribed papules that varied in size and number (41). These tumors appeared during the second decade of life. Through genomic sequencing of the melanocytic tumor specimens, a frameshift mutation in BAP1 was identified (41). The term melanocytic BAP1-MBAIT has been previously coined to describe these skin lesions $(3,33)$. The majority of familial epithelioid Spitz tumors or MBAITs have biallelic loss of $B A P 1$ via $B A P 1$ germline mutation, $3 \mathrm{p} 21.1$ chromosomal deletion, or uniparental disomy (7).

Cutaneous melanoma develops in approximately onethird of individuals with germline BAP1 loss (41). The role of BAP1 in melanocytic neoplasia depends on the presence of associated oncogenes (41). The median age at diagnosis of cutaneous melanoma was 41 years in a cohort of 38 patients with germline BAP1 mutations (21). The estimated penetrance of MBAITs and cutaneous melanoma in patients with germline $B A P 1$ mutations was reported to be $17 \%$ and $18 \%$, respectively, although may be as high as $75 \%$ (21).

\section{Suggested screening tests for cutaneous melanoma in germline BAP1 mutated individuals (34)}

Perform biannual dermatologic examinations by a melanoma specialist, including full-skin examination with systematic review of total body photography (TBP) starting at 18 years. Sequential digital dermatoscopic monitoring or excision of any mildly irregular skin lesion can be considered.

\section{Renal cell carcinoma}

RCC due to inheritable genetic mutations may account for $2-4 \%$ of cases (42). Mutations in genes involved in the ubiquitin-mediated proteolysis pathway (UMPP) activating the hypoxia regulatory network were found in $50 \%$ of ccRCC cases (43). Somatic inactivation of BAP1 has been demonstrated in $8-14 \%$ of ccRCCs $(11,43)$. BAP1 functions as a two-hit tumor suppressor in ccRCC and no second hit point mutations or deletions were found in tumors with $B A P 1$ mutations (11). This is in opposition to $M M$ and cutaneous melanoma studies which show loss of both $B A P 1$ alleles $(39,44)$. An association between high tumor grade and mTORC1 activation has been linked to $B A P 1$ loss (11). One study of families with germline mutations in BAP1 showed 6 of 11 families were affected by ccRCC (42). Nine total cases of ccRCC were detected in these families, suggesting mutated $B A P 1$ plays a role in ccRCC oncogenesis and may increase risk of ccRCC by 8 -fold (42).

\section{Suggested screening tests for RCC in germline BAP1 mutated individuals (34)}

Recommend annual abdominal physical examinations starting at age 30. Imaging surveillance using US or MRI can be considered for asymptomatic patients at age 30-55 every 2 years. After 55 years of age, perform CT chest/ abdomen or MRI chest/abdomen every 2 years, both with IV contrast.

\section{Other malignancies}

Among patients with meningiomas with rhabdoid features, $10 \%$ were found to have loss of BAP1 by immunohistochemistry (45). Two of these patients had detectable germline mutations in $B A P 1$, one with a splice site mutation and the other with a nonsense mutation. Other histologic features in this cohort of patients included sheets of dedifferentiated cells and epithelioid-type cells with a papillary growth pattern (45). Meningiomas with germline loss of BAP1 followed a more aggressive clinical course compared to WT tumors (45). A majority of meningiomas with $B A P 1$ inactivation were classified as WHO grade $2-3$.

Jensen and colleagues showed two non-small cell lung cancer (NSCLC) cell lines had undetectable levels of BAP1 expression, suggesting that BAP1 may be a target 
for inactivation or down-regulation during NSCLC pathogenesis (1). In a family with known truncating germline mutation of $B A P 1$, lung adenocarcinoma was diagnosed in the proband (40). Other tumors that were reported to be associated with germline BAP1 mutations include breast cancer, ovarian cancer non-melanoma skin cancer, cholangiocarcinoma, leiomyosarcoma, neuroendocrine cancer, pancreatic cancer, and paragangliomas (3).

\section{Treatment implications}

Clinical research continues to establish treatment implications for patients with $B A P 1$ loss, whether somatic or germline. Histone deacetylase (HDAC) inhibitors reversed the H2A hyperubiquitination caused by BAP1 loss, and they shifted the gene expression profile of class 2 cells toward a class 1 profile in a UVM cell line (44). HDAC inhibitors, thus, may slow the rate of metastatic spread of UVM cells and change the disease to a less aggressive phenotype (44). A phase 3 study comparing vorinostat (a HDAC inhibitor) with placebo in relapsed or refractory MPM concluded vorinostat did not improve OS compared to placebo and led to a statistically significant but not clinically relevant improvement in PFS (46). Molecular analysis to detect $B A P 1$ mutations in patients treated on this study has not been reported (46). BAP1 loss leads to increased expression of EZH2 (15). EZH2 inhibitors are a potential therapeutic strategy for BAP1-deficient tumors as mentioned previously.

PARP inhibition is another potential targeted therapy option in patients with somatic or germline BAP1 mutations. Clinical trials are underway to investigate the role of PARP inhibitors in patients with DNA-repair protein defects, including BAP1. Currently there is one trial of niraparib (PARP inhibitor) (Clinicaltrials.gov ID NCT03207347) and three trials investigating olaparib (another PARP inhibitor) in BAP1 and other DDR deficient neoplasms (Clinicaltrials. gov ID NCT03786796, NCT03531840, NCT03375307).

A recent study published by Hassan $e t$ al. suggested that patients with pleural mesothelioma with loss-of-function mutations in BAP1 and other DNA repair genes appeared to benefit from platinum chemotherapy compared with patients without inherited mutations (47).

\section{Conclusions}

Physicians and other healthcare professionals caring for patients with mesothelioma should be educated about BAP1-TPDS and potential alarming symptoms in BAP1 germline mutation carriers that require evaluation by a specialty or multidisciplinary clinic. Educating BAP1 germline mutation carriers and their family members is equally important. Particular attention should be given to symptoms including anorexia, weight loss, abdominal pain, respiratory symptoms, visual symptoms, and new or changing skin lesions. Patients should be educated on general cancer prevention strategies like avoiding asbestos and tobacco exposure as well as applying sunscreen before going outdoors. There are no established guidelines for screening patients for germline BAP1 mutations. Family predisposition to rare cancers such as UM or MM should raise the concern for an underlying germline $B A P 1$ and should be taken into consideration when making individualized screening recommendations.

Identifying individuals with $B A P 1$ germline mutation may trigger further testing in other family member to identify other at-risk family members. Germline testing should be considered in the context of genetic counseling. Children of individuals with germline BAP1 mutations should consider testing at age 18 to facilitate appropriate cancer screening. Testing in younger individuals may require more complex decision making that should take into account the ethical framework of autonomy, justice, beneficence and maleficence. Finally, there are currently no specific therapies approved by the FDA for treatment of $B A P 1$ mutant carriers with cancer, and this is an ongoing area of active research.

\section{Acknowledgments}

Funding: None.

\section{Footnote}

Conflicts of Interest: The authors have no conflicts of interest to declare.

Ethical Statement: The authors are accountable for all aspects of the work in ensuring that questions related to the accuracy or integrity of any part of the work are appropriately investigated and resolved.

Open Access Statement: This is an Open Access article distributed in accordance with the Creative Commons Attribution-NonCommercial-NoDerivs 4.0 International License (CC BY-NC-ND 4.0), which permits the noncommercial replication and distribution of the article with 
the strict proviso that no changes or edits are made and the original work is properly cited (including links to both the formal publication through the relevant DOI and the license). See: https://creativecommons.org/licenses/by-ncnd/4.0/.

\section{References}

1. Jensen DE, Proctor M, Marquis ST, et al. BAP1: a novel ubiquitin hydrolase which binds to the BRCA1 RING finger and enhances BRCA1-mediated cell growth suppression. Oncogene 1998;16:1097-112.

2. White AE, Harper JW. Cancer. Emerging anatomy of the BAP1 tumor suppressor system. Science 2012;337:1463-4.

3. Carbone M, Yang H, Pass HI, et al. BAP1 and cancer. Nat Rev Cancer 2013;13:153-9.

4. Bononi A, Giorgi C, Patergnani S, et al. BAP1 regulates IP3R3-mediated Ca2+ flux to mitochondria suppressing cell transformation. Nature 2017;546:549-53.

5. Bononi A, Yang H, Giorgi C, et al. Germline BAP1 mutations induce a Warburg effect. Cell Death Differ 2017;24:1694-704.

6. Carbone M, Flores EG, Emi M, et al. Combined genetic and genealogic studies uncover a large BAP1 cancer syndrome kindred tracing back nine generations to a common ancestor from the 1700s. PLoS Genet 2015;11:e1005633.

7. Murali R, Wiesner T, Scolyer RA. Tumours associated with BAP1 mutations. Pathology 2013;45:116-26.

8. Dey A, Seshasayee D, Noubade R, et al. Loss of the tumor suppressor BAP1 causes myeloid transformation. Science 2012;337:1541-6.

9. Stokes MP, Rush J, Macneill J, et al. Profiling of UVinduced ATM/ATR signaling pathways. Proc Natl Acad Sci U S A 2007;104:19855-60.

10. Ismail IH, Davidson R, Gagné JP, et al. Germline mutations in BAP1 impair its function in DNA doublestrand break repair. Cancer Res 2014;74:4282-94.

11. Peña-Llopis S, Vega-Rubín-de-Celis S, Liao A, et al. BAP1 loss defines a new class of renal cell carcinoma. Nat Genet 2012;44:751-9.

12. Parrotta R, Okonska A, Ronner M, et al. A novel BRCA1-associated protein-1 isoform affects response of mesothelioma cells to drugs impairing BRCA1-mediated DNA repair. J Thorac Oncol 2017;12:1309-19.

13. Margueron R, Reinberg D. The Polycomb complex PRC2 and its mark in life. Nature 2011;469:343-9.

14. Yamagishi M, Uchimaru K. Targeting EZH2 in cancer therapy. Curr Opin Oncol 2017;29:375-81.

15. LaFave LM, Béguelin W, Koche R, et al. Loss of BAP1 function leads to EZH2-dependent transformation. Nat Med 2015;21:1344-9.

16. Schoumacher M, Le Corre S, Houy A, et al. Uveal melanoma cells are resistant to EZH2 inhibition regardless of BAP1 status. Nat Med 2016;22:577-8.

17. Zauderer MG, Szlosarek P, Le Moulec S, et al. Phase 2, multicenter study of the EZH2 inhibitor tazemetostat as monotherapy in adults with relapsed or refractory (R/R) malignant mesothelioma (MM) with BAP1 inactivation. J Clin Oncol 2018;36:8515.

18. Warburg O. On the origin of cancer cells. Science 1956;123:309-14.

19. Cairns RA, Harris IS, Mak TW. Regulation of cancer cell metabolism. Nat Rev Cancer 2011;11:85-95.

20. Testa JR, Cheung M, Pei J, et al. Germline BAP1 mutations predispose to malignant mesothelioma. Nat Genet 2011;43:1022-5.

21. Haugh AM, Njauw CN, Bubley JA, et al. Genotypic and phenotypic features of BAP1 cancer syndrome: a report of 8 new families and review of cases in the literature. JAMA Dermatol 2017;153:999-1006.

22. Pastorino S, Yoshikawa Y, Pass HI, et al. A subset of mesotheliomas with improved survival occurring in carriers of BAP1 and other germline mutations. J Clin Oncol 2018. [Epub ahead of print].

23. Rai K, Pilarski R, Cebulla CM, et al. Comprehensive review of BAP1 tumor predisposition syndrome with report of two new cases. Clin Genet 2016;89:285-94.

24. Kadariya Y, Cheung $\mathrm{M}, \mathrm{Xu} \mathrm{J}$, et al. Bap1 is a bona fide tumor suppressor: genetic evidence from mouse models carrying heterozygous germline Bap1 mutations. Cancer Res 2016;76:2836-44.

25. Delgermaa V, Takahashi K, Park EK, et al. Global mesothelioma deaths reported to the World Health Organization between 1994 and 2008. Bull World Health Organ 2011;89:716-24, 724A-724C.

26. Carbone M, Adusumilli PS, Alexander HR Jr, et al. Mesothelioma: scientific clues for prevention, diagnosis, and therapy. CA Cancer J Clin 2019;69:402-29.

27. Bott M, Brevet M, Taylor BS, et al. The nuclear deubiquitinase BAP1 is commonly inactivated by somatic mutations and 3p21.1 losses in malignant pleural mesothelioma. Nat Genet 2011;43:668-72.

28. Ohar JA, Cheung M, Talarchek J, et al. Germline BAP1 mutational landscape of asbestos-exposed malignant mesothelioma patients with family history of cancer. 
Cancer Res 2016;76:206-15.

29. Panou V, Gadiraju M, Wolin A, et al. Frequency of germline mutations in cancer susceptibility genes in malignant mesothelioma. J Clin Oncol 2018;36:2863-71.

30. Baumann F, Flores E, Napolitano A, et al. Mesothelioma patients with germline BAP1 mutations have 7-fold improved long-term survival. Carcinogenesis 2015;36:76-81.

31. Xu J, Kadariya Y, Cheung M, et al. Germline mutation of Bap1 accelerates development of asbestos-induced malignant mesothelioma. Cancer Res 2014;74:4388-97.

32. Napolitano A, Pellegrini L, Dey A, et al. Minimal asbestos exposure in germline BAP1 heterozygous mice is associated with deregulated inflammatory response and increased risk of mesothelioma. Oncogene 2016;35:1996-2002.

33. Carbone M, Ferris LK, Baumann F, et al. BAP1 cancer syndrome: malignant mesothelioma, uveal and cutaneous melanoma, and MBAITs. J Transl Med 2012;10:179.

34. Star P, Goodwin A, Kapoor R, et al. Germline BAP1positive patients: the dilemmas of cancer surveillance and a proposed interdisciplinary consensus monitoring strategy. Eur J Cancer 2018;92:48-53.

35. Carbone M, Kanodia S, Chao A, et al. Consensus report of the 2015 Weinman international conference on mesothelioma. J Thorac Oncol 2016;11:1246-62.

36. Kittaneh M, Berkelhammer C. Detecting germline BAP1 mutations in patients with peritoneal mesothelioma: benefits to patient and family members. J Transl Med 2018;16:194.

37. Onken MD, Worley LA, Tuscan MD, et al. An accurate, clinically feasible multi-gene expression assay for predicting metastasis in uveal melanoma. J Mol Diagn 2010;12:461-8.

38. Chang SH, Worley LA, Onken MD, et al. Prognostic biomarkers in uveal melanoma: evidence for a stem cell-

Cite this article as: Kobrinski DA, Yang H, Kittaneh M. BAP1: role in carcinogenesis and clinical implications. Transl Lung Cancer Res 2020;9(Suppl 1):S60-S66. doi: 10.21037/ tlcr.2019.11.24 like phenotype associated with metastasis. Melanoma Res 2008;18:191-200.

39. Harbour JW, Onken MD, Roberson ED, et al. Frequent mutation of BAP1 in metastasizing uveal melanomas. Science 2010;330:1410-3.

40. Abdel-Rahman MH, Pilarski R, Cebulla CM, et al. Germline BAP1 mutation predisposes to uveal melanoma, lung adenocarcinoma, meningioma, and other cancers. J Med Genet 2011;48:856-9.

41. Wiesner T, Obenauf AC, Murali R, et al. Germline mutations in BAP1 predispose to melanocytic tumors. Nat Genet 2011;43:1018-21.

42. Popova T, Hebert L, Jacquemin V, et al. Germline BAP1 mutations predispose to renal cell carcinomas. Am J Hum Genet 2013;92:974-80.

43. Guo G, Gui Y, Gao S, et al. Frequent mutations of genes encoding ubiquitin-mediated proteolysis pathway components in clear cell renal cell carcinoma. Nat Genet 2011;44:17-9.

44. Landreville S, Agapova OA, Matatall KA, et al. Histone deacetylase inhibitors induce growth arrest and differentiation in uveal melanoma. Clin Cancer Res 2012;18:408-16.

45. Shankar GM, Santagata S. BAP1 mutations in high-grade meningioma: implications for patient care. Neuro Oncol 2017;19:1447-56.

46. Krug LM, Kindler HL, Calvert H, et al. Vorinostat in patients with advanced malignant pleural mesothelioma who have progressed on previous chemotherapy (VANTAGE-014): a phase 3, double-blind, randomised, placebo-controlled trial. Lancet Oncol 2015;16:447-56.

47. Hassan R, Morrow B, Thomas A, et al. Inherited predisposition to malignant mesothelioma and overall survival following platinum chemotherapy. Proc Natl Acad Sci U S A 2019;116:9008-13. 